\title{
Effect of Dual Task Training in Visual Control and Unstable Base on the Gait of Stroke Patient
}

\begin{abstract}
This study examines changes in walking ability among patients with stroke after applying dual-task training under the condition of visual control and unstable supporting ground; the purpose is to provide reference data for selecting intervention methods that enhance the walking ability of patients with stroke. Among the patients with stroke who received rehabilitation treatment(at Rehabilitation Hospital B in Gyeonggi, South Korea from May 2014 to July 2014), 29 patients were selected as research subjects; all of them understood the purpose and contents of this research and agreed to participate in the experiment. The research subjects were divided into a visual control and unstable supporting ground dual-task(VUDT) group(10 patients), a visual control dual-task(VDT) group(10 patients), and an unstable supporting ground dual-task(UDT) group(9 patients); all of the subjects received 30-minute trainings, three times a week for a total of four weeks. A Timed-Up-and-Go(TUG) test was performed to investigate the change of walking function among the subjects, and a $10 \mathrm{~m}$ walking test was conducted to measure their walking speed. According to the study results, all three groups showed significant differences after dual-task training; the dual-task training group under the condition of visual control and unstable supporting ground showed the most prominent change. This study confirmed that dual-task training using visual control and unstable supporting ground has a positive impact on the walking ability of patients with stroke. Through the study results, we found that implementing dual-task training under the condition of visual control and unstable supporting ground can more effectively improve the walking ability of patients with stroke, rather than performing visual control dual-task training or unstable supporting ground dual-task training only.
\end{abstract}

Key words: Walking; Stroke; Dual-task training

\author{
Sa Gyeom Lee ${ }^{a}$, Yang Rae Kim ${ }^{\mathrm{b}}$ \\ aSuwon Women's University, Suwon; 'Anyang \\ Sam Hospital, Anyang, Korea
}

Received : 4 December 2014

Revised : 10 January 2015

Accepted : 27 February 2015

\section{Address for correspondence}

Sa Gyeom Lee, PT, Ph.D

Department of Physical Therapy, Suwon Women's College, 336-27 Sanggi-ri, Bongdam-eup, Hwaseong, Korea

Tel: 82-31-290-8956

E-mail: sglee@swc.ac.kr

*This paper was funded by 2014 research supported by suwon women's University.

\section{INTRODUCTION}

Stroke is a disease where partial loss of brain function occurs due to ischemia or hemorrhage; it induces a number of functional disabilities, such as muscle strength weakening, rigidity, pain, and balancing disorder(1). Among these, the occurrence of balancing disorder is caused by loss of ability to harmoniously use vestibular sense, somesthesia, proprioceptive sense, etc., thus diminishing the capacity of the central nervous system(which integrates the received sensing information with the motor system), and leading to abnormal tonus of muscle(2, 3).

The ability to move weight in diverse postures and directions is important for performing daily activities. Patients with stroke face an increased risk of falling due to asymmetrical weight bearing; difficulties in walking limits their performance of daily activities $(4,5,6,7)$.

For normal walking, it is necessary to have the ability to symmetrically distribute weight bearing on the less affected side's lower limb, and the more affected side's lower limb during the standing 
position. Allowing hemiparalysis patients to have weight bearing on the affected side's lower limb makes them perceive their legs, improves their sensory function, normalizes the muscle stiffness, and decreases spasticity.

Patients with stroke have diminished balancing ability due to the biased standing on the less affected side's lower limb(8), and they experience increased swaying in the static standing position (9). Moreover, patients with stroke show loss of ability to control the central nervous system, as well as excessive tonus of muscle, such as rigidity. In addition, they tend to experience incongruity of movement and antagonism, and the loss of proprioceptive sense and balancing sense(10). This makes it hard for them to control normal posture, which is an important reason for diminished walking ability(11, 12).

Vision is the sensory input that posture control depends upon most, and it plays the most important role in controlling posture(13). Since we can stand with our eyes closed or we can stand still in a dark room, however, posture control does not necessarily require vision. Moreover, visual information can be misinterpreted by our brains. The visual system has difficulty in determining whether the surroundings are moving or the person himself/herself is moving. Hence, not only the visual sensor, but also the somatic sensor and the vestibular system are important in posture control in a standing position(14). Meanwhile, patients with stroke tend to overly depend on vision, and such excessive visual dependence eventually causes posture imbalance(15).

According to Shumway-Cook and Wollacott(16), posture control depends on the somatosensory information coming from the foot that contacts the supporting ground. If external disturbance is increased by providing unstable supporting ground, postural orientation ability effectively changes and the sensory and motor system become capable of more prompt correction, which helps the postural strategy for posture control. Unstable supporting ground and stable supporting ground provoke different somatic sensors. Hence, an effort to maintain standing balance on an unstable supporting ground itself helps to develop diverse reaction forces, activating all of the tendon, ligament, and joint receptors(17).

The recovery of independent balancing and walking ability after a stroke is a common goal of patients and therapists(18). A number of previous studies have aimed to develop intervention meth- ods for improving the balancing and walking abilities of patients with stroke(19, 20, 21).

"Dual-task training" refers to a training regime where a subject performs one task while performing another one, or continuously performs two or more tasks(22). Research on dual-task training can be largely divided into two groups. The first is "dual cognition task training," where a subject performs cognition tasks or mental tasks(such as answering questions, calculating numbers, or suggesting vocabularies) while walking, or maintaining a standing position at the same time(23). The interaction between posture control and attention and cognition during this process was investigated by Muhaidat et al.(24). The second group includes choosing objects while walking on a treadmill(25) or moving plates and cups during walking tasks $(26,27)$. In these dual-exercise task trainings, the changes in walking speed and stride length during walking are examined in order to investigate the patients' functional recovery and prognosis. As such, dual-task research is used to focus on the evaluation of balance and posture control of patients with neurological damage, and the verification of the effectiveness of walking training(28).

However, research on the balancing and walking ability of patients with stroke that simultaneously considers visual control and supporting ground conditions in performing dual-task exercises related to posture control factors is very rare as of now. In order to enhance the effects of dual-task training, visual influence(29) and supporting ground influence(30) are very important.

Hence, this study implemented dual-task training that simultaneously considers visual control and supporting ground conditions on patients with stroke for the purpose of investigating its impact on walking ability.

\section{METHODS}

\section{Subjects}

Among the patients with stroke who received rehabilitation treatment at Rehabilitation Hospital B in Gyeonggi-do, South Korea during the period from May 2014 to July 2014, 29 patients were selected; all patients understood the purpose and contents of this research and agreed to participate in the experiment. The research subjects were divided into a visual control and unstable supporting 
ground dual-task(VUDT) group, a visual control dual-task(VDT) group, and an unstable supporting ground dual-task(UDT) group. These groups had 10, 10, and 9 subjects, respectively; they each received 30-minute trainings, three times a week for eight weeks〈Table 1〉.

The VUDT group performed a training in which they stood on stable ground and carried out cognition tasks while maintaining posture after removing visual information by covering their eyes with eye patches, and a training where they performed a cognition task while maintaining balance on an unstable supporting ground(a balancing pad), each of which was performed for one set. The cognition tasks were implemented three times a week, as suggested by previous studies. In addition to this, conservative treatment was implemented, and the tasks were reformed such that the difficulty level matched the patients(31).

The VDT group performed a training in which they stood on stable ground and performed the previously mentioned cognition tasks while maintaining posture after removing visual information by covering their eyes with eye patches for two 15-minute sets.

The UDT group performed a training in which they carried out the previously mentioned cognition tasks while maintaining balance on an unstable supporting ground for two 15-minute sets.

The inclusion criteria for the research subjects included those who had received a stroke diagnosis more than six months previously; those who were capable of walking more than $100 \mathrm{~m}$; those without neurological disease such as amblyopia, dizziness, and vestibular function abnormalities; those with Mini-Mental State ExaminationKorea(MMSE-K) scores over 24; and those without orthopedic disease in the lower limbs.

Table 1. Cognition task components

\begin{tabular}{ll}
\hline & \multicolumn{1}{c}{ Cognition task program } \\
\hline & 1. Randomly speaking numbers \\
Day 1 2. Counting backward & $\begin{array}{l}\text { 3. Memorizing backward(number, months, day of } \\
\text { the week, etc.) }\end{array}$ \\
& 4. Memorizing objects \\
Day 2 5. Speaking objects or words & $\begin{array}{l}\text { 6. Completing sentences } \\
\text { Day 3 } 3\end{array}$ \\
& $\begin{array}{l}\text { 7. Speaking words backward } \\
\text { 9. Mimicking sentences }\end{array}$ \\
\hline
\end{tabular}

\section{Measurement tools}

\section{TUG}

The Timed-Up-and-Go(TUG) test is a test that measures functional mobility and balancing ability. In this study, the time was measured in which the research subjects sat down in an armchair, stood up at the tester's signal to walk $3 \mathrm{~m}$, and returned to sit down again. After practicing once, the subjects repeated the test three times, and the average value was used as the TUG performing time. The TUG test generally takes about 7-10 seconds for a healthy, normal elderly person on average; a time exceeding 30 seconds is known to indicate dependent moving ability or disability to independently carry out outdoor exercise(32). The TUG test reportedly has high reliability, with an intra-rater reliability of $\mathrm{r}=.99$ and inter-rater reliability of $r=.98(33)$.

\section{$10 \mathrm{~m}$ walking speed test}

A $10 \mathrm{~m}$ walking test was conducted in order to examine the subjects' walking speed. The subject walked a total $13 \mathrm{~m}$; their time to do so was measured using a stopwatch after excluding $1.5 \mathrm{~m}$ from each of the predetermined beginning points and arrival points. The experiment subjects received information about the test beforehand, and were told that they should walk at their ordinary walking speed until they arrived at the finishing point. Measuring walking speed within a $10 \mathrm{~m}$ distance is known to have high intra-rater reliability as well as inter-rater reliability $(\mathrm{r}=.89 \sim .100)$.

\section{Data analysis}

Collected data were processed using the PASW program(ver. 18.0) for data treatment and hypothesis testing according to the research purpose. First, a test of normality was performed on the total subjects' data, whose results indicated normal distribution of every variable. Second, for comparing the within-group and between-group difference after applying the dual-task training, Two-way Repeated Measure ANOVA was conducted. The statistical significance level was set at $\alpha<.05$ in this study.

\section{RESULTS}

The study examined the changes in walking ability 
after implementing dual-task training on patients with stroke under the conditions of visual control and unstable supporting ground.

1. General characteristics of the research subjects
2. Within-group and between-group comparison of the three groups in their walking ability before and after the training

Table 2. General characteristics of the research subjects

\begin{tabular}{|c|c|c|c|c|}
\hline Category & $\operatorname{VUDT}(n=10)$ & $\operatorname{VDT}(n=10)$ & $\operatorname{UDT}(n=9)$ & $\mathrm{F}$ \\
\hline Age & $55.70 \pm 11.92$ & $55.30 \pm 7.48$ & $52.56 \pm 11.43$ & .72 \\
\hline MMSE-K & $26.90 \pm 1.85$ & $26.50 \pm 1.78$ & $26.33 \pm 1.58$ & .68 \\
\hline \multicolumn{5}{|l|}{ Gender } \\
\hline Male & 5 & 4 & 5 & $x^{2}$ \\
\hline Female & 5 & 6 & 4 & .788 \\
\hline \multicolumn{5}{|l|}{ Diagnosis } \\
\hline Infarction & 6 & 4 & 5 & $x^{2}$ \\
\hline Hemorrhage & 4 & 6 & 4 & .645 \\
\hline \multicolumn{5}{|l|}{ Paralyzed side } \\
\hline Left & 6 & 4 & 6 & $x^{2}$ \\
\hline Right & 4 & 6 & 3 & .471 \\
\hline
\end{tabular}

Table 3. Within-group and between-group comparison in walking ability before and after the training

\begin{tabular}{|c|c|c|c|c|c|c|}
\hline Category & & VUDT $(n=10)$ & VDT $(n=10)$ & UDT $(n=9)$ & F & $\mathrm{p}$ \\
\hline \multirow{4}{*}{ TUG } & Before training & $21.90 \pm 12.70$ & $22.06 \pm 14.43$ & $18.20 \pm 7.77$ & \multirow{4}{*}{9.992} & \multirow{4}{*}{.001} \\
\hline & After training & $17.96 \pm 11.70$ & $19.83 \pm 13.96$ & $16.03 \pm 7.72$ & & \\
\hline & Change & $-3.94 \pm 1.51$ & $-2.23 \pm .70$ & $-2.17 \pm .31$ & & \\
\hline & $p$ & .000 & .000 & .000 & & \\
\hline \multirow{4}{*}{$10 \mathrm{~m}$ gait velocity } & Before training & $19.15 \pm 8.58$ & $23.31 \pm 19.03$ & $19.21 \pm 10.64$ & \multirow{4}{*}{11.862} & \multirow{4}{*}{.000} \\
\hline & After training & $14.95 \pm 7.16$ & $20.45 \pm 18.33$ & $17.53 \pm 10.27$ & & \\
\hline & Change & $-4.20 \pm 1.60$ & $-2.86 \pm .93$ & $-1.68 \pm .54$ & & \\
\hline & $\mathrm{p}$ & .000 & .000 & .000 & & \\
\hline
\end{tabular}

All of the walking ability items showed significant differences after the training in each of the three groups $(p<.05)$. Among them, the VUDT group showed the largest improvement. As for the between-group difference, the VUDT group showed significant improvement in both the TUG test and $10 \mathrm{~m}$ walking speed( $\mathrm{p}<.05)$. Table 2 pres- $^{-}$ ents the within-group and between-group comparisons of the three groups in their walking ability $\langle$ Table 3$\rangle$.

\section{DISCUSSION}

According to the experiment results, which examined walking ability by applying dual-task training to patients with stroke under the condition of visual control and unstable supporting ground, walking ability improvement was observed in all three groups; among these, the VUDT group showed the most prominent enhancement.

Bowen et al.(23) reported that walking speed decreased when subjects performed secondary tasks while walking at a steady speed. Silsupadol 
et al.(31) reported, in an experiment with a sample of elderly people who had balancing damage, that walking speed decreased in training groups that had received dual tasks under three different conditions. Kizony et al.(25) conducted an experiment where a single-task training group performed a single-task training of walking on a treadmill, while an experiment group performed dual-task training of walking on a treadmill and selecting objects through the screen in a virtual reality environment. They showed that the single-task walking group showed improved walking speed, while the dual-task training group showed improvement in both walking speed and stride length. Marigold and Eng(34) conducted an experiment on 28 patients with stroke and 28 people without stroke to investigate the visual dependence of the stroke patients. The study reported that the patients with stroke excessively depended on vision; the authors argued that the task of closing the eyes has the potential for improving balance among patients with stroke. Walker et al. (35) argued that there is a correlation between walking speed and TUG score. Considering that TUG test scores also increased in this study, we conjecture that walking ability was improved. Haart et al.(36) reported that the provision of inner- and outer postural sway to patients with stroke by using a dual-plate force platform not only reinforces standing balance, but also has a positive impact on recovery. Matsusaka et al.(37) implemented balancing disk training combined with tactile stimulation on patients with functional ankle joint instability, and found that the time required for the body swaying degree to be normalized decreased. Irion(38) argued that a training on unstable supporting ground better stimulates posture control and dynamic balancing compared to training on stable supporting ground.

Similarly to these previous studies' results, the significant difference observed in the dual-task training group in this study can be attributed to the task training related to the body trunk control and active control of muscles that effectively improves walking factors in a positive direction following the intervention period(39). We believe the active reinforcement of lower limb muscles through diverse control of muscle activities caused the significant difference in certain walking variables. Moreover, although every group showed significant differences after dual-task training, the dual-task training group under the condition of visual control and unstable supporting ground showed the most prominent change. Future research on functional walking evaluations that can examine the qualitative change of walking will be meaningful.

This study confirmed that dual-task training that uses visual control and unstable supporting ground affects the walking ability of patients with stroke. We conjecture that dual-task training that incorporates cognition tasks not only improves the balance and walking ability of patients with chronic stroke, but also enhances their cognitive ability.

This study did have some limitations. The eightweek research period for investigating the training effects was relatively short, and the lack of a follow-up study means that we were unable to know how long the effects lasted. In addition, the study could not sufficiently control for the dual tasks or mobility that the subjects carried out in the course of their daily routines, and the sample size was not large enough to generalize the study results to all patients with stroke.

\section{CONCLUSION}

This study confirmed that dual-task training that uses visual control and unstable supporting ground has a positive impact on the walking ability of patients with stroke. Through the study results, we found that implementing training under the conditions of visual control and unstable supporting ground can more effectively improve the walking ability of patients with stroke in the future, rather than performing visual control dual-task training or unstable supporting ground dual-task training only.

\section{REFERENCES}

1. Prange GB, Jannink MJ, Groothuis-Oudshoorn CG, et al. Systematic review of the effect of robotaided therapy on recovery of the hemiparetic arm after stroke. J Rehabil Res Dev. 2006; 43(2): 171184.

2. Mercier L, Audet T, Hébert R, et al. Impact of motor, cognitive, and perceptual disorders on ability to perform activities of daily living after stroke. Stroke. 2001; 32(11): 2602-2608. 
3. Shumway-Cook A, Anson D, Haller S. Postural sway biofeedback: Its effect on reestablishing stance stability in hemiplegic patients. Arch Phys Med Rehabil. 1988; 69(6): 395-400.

4. Chen G, Patten C. Treadmill training with harness support: Selection of parameters for individuals with poststroke hemiparesis. J Rehabil Res Dev. 2006; 43(4): 485-498.

5. Eng JJ, Chu KS. Reliability and comparison of weight-bearing ability during standing tasks for individuals with chronic stroke. Arch Phys Med Rehabil. 2002; 83(8): 1138-1144.

6. Forster A, Young J. Incidence and consequences of falls due to stroke: A systematic inquiry. BMJ. 1995; 311(6997): 83-86.

7. Laufer Y, Dickstein R, Resnik S, et al. Weightbearing shifts of hemiparetic and healthy adults upon stepping on stairs of various heights. Clin Rehabil. 2000; 14(2): 125-129.

8. Nyberg L, Gustafson Y. Patient fall in stroke rehabilitation. Stroke. 1995; 26(1): 838-842.

9. Dickstein R, Abulaffio N. Postural sway of the affected and non affected pelvis and leg instance of hemiparetic patients. Arch Phys Med Rehabil. 2000; 81(3): 364-367.

10. Kerrigan DC, Karvosky EM, Riley PO. Spastic paretic stiff-legged gait: Joint kinetics. Arch Phys Med Rehabil. 2001; 80(4): 244-249.

11. Carr J, Shepherd R. Stroke rehabilitation: Guidelines for exercise and training to optimize motor skill. Oxford: Elsevier, 2004.

12. Varoqui D, Froger J, Lagarde J, et al. Changes in preferred postural patterns following stroke during intentional ankle/hip coordination. Gait and Posture. 2010; 32(1): 34-38.

13. Massion J, Amblard B, Assaiante C, et al. Body orientation and control of coordinated movements in microgravity. Brain Res Brain Res Rev. 1998; 28(1): 83-91.

14. Shumway-Cook A, Wollacott M. Attentional demands and postural control: The effect of sensory context. J Gerontol A Biol Sci Med Sci. 2000; 55(1): 245-356.

15. Bonan IV, Yelnik AP, Colle FM, et al. Reliance on visual information after stroke. Part II: Effectiveness of a balance rehabilitation program with visual cue deprivation after stroke. A randomized controlled trial. Arch Phys Med Rehabil. 2004; 85( 2): 274-278.

16. Shumway-Cook A, Woollacott MH. Motor control: Translating research into clinical practice. 3rd ed. Philadelphia, Lippincott Williams \& Wilkins, 2007; 3-83.
17. Lee SH. The differences between aero step exercises and weight training on posture, physical fitness, balance, and hormone levels in the elderly. Ewha Womans University, master' s thesis, 2007.

18. Maguire C, Sieben JM, Frank M, et al. Hip abductor control in walking following stroke the immediate effect of canes, taping and theratogs on gait. Clin Rehabil. 2010; 24(1): 37-45.

19. Ada L, Dean CM, Hall JM, et al. A treadmill and overground walking program improves walking in persons residing in the community after stroke: A placebo-controlled, randomized trial. Arch Phys Med Rehabil. 2003; 84(10): 1486-1491.

20. Kim JJ. Influence of side walking on gait and weight bearing in hemiplegic patients with a stroke. Yongin, DanKook University, Master Thesis. 2000.

21. Yelnik AP, Le Breton F, Colle FM, et al. Rehabilitation of balance after stroke with multisensorial training: A single-blind randomized controlled study. Neurorehabil Neural Repair. 2008; 22(5): 468-476.

22. Pellecchia GL, Shockley K, Turvey MT. Concurrent cognitive task modulates coordination dynamics. Cognitive Science. 2005; 29(4): 531-557.

23. Bowen A, Wenman R, Mickelborough J, et al. Dual-task effects of talking while walking on velocity and balance following a stroke. Ages Ageing. 2001; 30(4): 319-23.

24. Muhaidat J, Skelton DA, Kerr A, et al. 182 are we using the right dual tasks to detect mobility problems? perspectives from older people. Gait \& Mental Function. 2010; 16(1): S52-S53.

25. Kizony R, Levin MF, Hughey L et al. Cognitive load and dual-task performance during locamotion poststroke: A feasibility study using a functional vitual environment. Phys Ther. 2010; 90(2): 252-260.

26. Yang YR, Chung YC, Lee CS et al. Dual taskrelated gait changes in individuals with stroke. Gait and Posture. 2007; 25(2): 185-90.

27. Melzer I, Tzedek I, Or M et al. Speed of voluntary stepping in chronic stroke survivors under single-task and dual-task conditions: A case-control study. Electronic. 2009; 90(6): 927-33.

28. Alain L, Héelèene P, \& Sylvie N. Task-oriented intervention in chronic stroke. Am J Phys Med Rehabil , 2006; 85(10): 820-830.

29. Bensoussan L, Viton JM, Schieppati M, et al. Changes in postural control in hemiplegic patients after stroke performing a dual task. Arch Phys Med Rehabil. 2007; 88(8):1 009-15. 
30. Her, JK, Park, KD, Yang, YE. Effect of balance training with various patients: dual-task conditions on stroke. J Phys Ther Sci. 2011; 23(5): 713717.

31. Silsupadol P, Lugade V, Shumway-Cook A et al. Training-related changes in dual-task walking performance of elderly persons with balance impairment: A double-blind, randomized controlled trial. Gait and Posture. 2009; 29(4): 634639.

32. Faria CD, Teixeira-Salmela LF, Nadeau S. Effects of the direction of turning on the timed up \& go test with stroke subjects. Top Stroke Rehabil. 2009; 16(3): 196-206.

33. Podsiadlo D, Richardson S. The timed "up \& go": A test of basic functional mobility for frail elderly persons. J Am Geriatr Soc. 1991; 39(2): 142-148. Marigold DS, Eng J. The relationship of asym-

34. metric weight-bearing with postural sway and visual reliance in stroke. Gait and Posture. 2005; 23( 2): 249-255.
35. Walker C, Brouwer BJ, Culham EG. Use of visual feedback in retraining balance following acute stroke. Phys Ther. 2000; 80(9): 886-895.

36. Haart M, Geurts AC, Huidekoper SC. Recovery of standing balance in postacute stroke patients: A rehabilitation cohort study. Arch Phys Med Rehabil. 2004; 85( 6): 886-895.

37. Matsusaka N, Yokoyama S, Tsursaki T, et al. Effects of ankle disk training combined with tactile stimulation to the leg and foot on functional instability of the ankle. Am J Sports Med. 2001; 29(1): 25-30.

38. Irion JM. Use of the zymball in rehabilitation of spinal dysfunction, J Am Orthopaedic Phys Ther Clin. 1992; 1(2): 375-398.

39. Dean CM, Carol LR, Fransine M. Task-related circuit training improves performance of locomotor tasks in chronic stroke: A randomized, controlled pilot trial. Physical Therapy. 2000; 81(4): 409-17. 